\title{
Correction to "Developmental Changes in Hepatic Organic Cation Transporter OCT1 Protein Expression from Neonates to Children"
}

\begin{abstract}
In the above article [Hahn D, Emoto C, Vinks AA, and Fukuda T (2017) Drug Metab Dispos 45(1):23-26; DOI: 10.1124/dmd.116.072256], NIH funding information was omitted. The funding footnote in total should read:

This research was supported in part by a T1 grant from the University of Cincinnati Center for Clinical and Translational Science and Training and the National Institutes of Health National Center for Advancing Translational Sciences [Award UL1 TR001425]. Support for the Better Outcomes for Children Biorepository is provided by the Cincinnati Children's Research Foundation of Children's Hospital Medical Center. Support for the Cincinnati Biobank is provided by the Children's Hospital Medical Center in conjunction with the University of Cincinnati.
\end{abstract}

The authors regret this error and any inconvenience it may have caused. 